separation of science into constituent parts, while there is ultimately a branching into the many distinct sciences. The troublesome problem of the closer relation of pure mathematics to its applications : can it not be solved by indirection, in that through the whole course of elementary mathematics, including the introduction to the calculus, there be recognized in the organization of the curriculum no distinction between the various branches of pure mathematics and likewise no distinction between pure mathematics and its principal applications? Further, from the standpoint of pure mathematics : will not the twentieth century find it possible to give to young students during their impressionable years in thoroughly concrete and captivating form the wonderful new notions of the seventeenth century? By way of suggestion these questions have been answered in the affirmative, on condition that there be established a thoroughgoing laboratory system of instruction in primary schools, secondary schools, and junior colleges - a laboratory system involving a synthesis and development of the best pedagogic methods at present in use in mathematics and the physical sciences.

\title{
CONCERNING THE AXIOM OF INFINITY AND MATHEMATICAL INDUCTION.
}

BY PROFESSOR C. J. KEYSER.

(Read before the American Mathematical Society, December 29, 1902.)

\section{Introductory Considerations.}

This paper deals with a question which, on the one hand, is a question of pure logic, and, on the other, a question of Mengenlehre. It is often asserted, and is probably true, that reasoning naturally takes place in accordance with what the logicians of the school called first intentions. But ratiocination as activity, however unconscious its conformation to law, is nevertheless not lawless; and from the period when this fact came clearly into the consciousness of the Greek mind, as early as the time of Protagoras,* science has been neither able nor

* The so-called laws of thought seem to have struggled into consciousness mainly through the disputations of the Sophists. The law of contradiction, in particular, appears to have received its earliest formulation in the $\kappa a \tau a \beta a ́ \lambda \lambda \nu \varepsilon \varepsilon$ of Protagoras. Cf. Windelband: Geschichte der Philosophie, and Ueberweg : System der Logik (both works also in English). 
willing to escape the consideration of second intentions in their logical significance. So true is this that, despite the age-long tyranny exercised by the Aristotelian logic - a tyranny having, at least in the domain of science, scarcely a match except in the case of Euclid's elements - the forms of thought, which serve as a kind of diagrammatic representation of the orderliness of the reasoning processes, sustain to-day perhaps even greater interest than ever before. The mathematician's interest in these forms is two-fold, attaching to them both as norms for mechanically testing the validity of arguments and as constituting exceedingly subtile matter for mathematical investigation.

Of all the argument forms, there is one which, viewed as the figure of the way in which the mind gains certainty that a specified property belongs to each element of a given assemblage, enjoys the distinction of being at once perhaps the most fascinating, and, in its mathematical bearings, doubtless the most important, single form in modern logic. I refer to the argument form variously known as reasoning by recurrence, induction by connection (De Morgan), mathematical induction, complete induction, and Fermatian * induction - a form of procedure unknown to the Aristotelean system, for this latter allows apodictic certainty in case of deduction only, while it is just the characteristic of complete induction that it yields such certainty by the reverse process, a movement from the particular to the general, from the finite to the infinite.

That the highest degree of certainty is thus attainable has been the living faith of mathematicians at least since the time of Fermat, and on it is based the whole modern movement towards the rigorization or, at all events, the arthmetization of mathematics, for, as is well known, it is precisely by means of complete induction that the fundamental laws of number have been or admit of being established for the totality of integers. It is accordingly not a matter for surprise that logicians, whether of the traditional or of the modern so-called " exact" $\dagger$ school, have felt challenged to examine the method in question and to seek an adequate a priori justification for the mentioned faith in its validity.

\footnotetext{
* So named by Mr. C. S. Peirce, according to whom this form of reasoning is due to Fermat.

† A minute analysis of the method in question, in connection with the presuppositions of Dedekind's proof of the "theorem of complete induction," is found in Schröder's Algebra der Logik, vol. 3.
} 
Among the discussions of the subject that are readily accessible to mathematicians not familiar with the symbology of technically mathematical logic, the most notable are that by Dedekind in his Was sind und Was sollen die Zahlen (also in English, 1901) and that by Poincaré in his article "Sur la nature du raisonnement mathématique" \$ (Revue de Métaphysique et de Morale, volume II). The latter essay, though written (1894) several years after the publication of the former, makes no allusion to it. The two discussions have in fact little in common save their problem, which is that of disclosing what the German calls "die wissenschaftliche Grundlage" of a mode of logical procedure characterized by the Frenchman as "le raisonnement mathématique par excellence." They approach their common task from the most widely sundered points of departure and along paths which conduct them finally to views that, as will subsequently appear, are neither coincident nor, strictly regarded, compatible. It is this striking difference of method and especially the essential though elusive difference of conclusion, which we plead as an excuse, or, at all events, as a sufficient provocation for undertaking to examine the matter once more. Such an examination will naturally involve a critical review and comparison of the discussions in question.

\section{Poincaré's View.-The Axiom of Infinity.}

For Poincare the question involved is of the farthest-reaching critical importance. To answer it is nothing less than to show not merely the logical validity but the logical possibility of mathematical science, because for such possibility it is necessary to be able to establish general theorems, $i$. e., to gain the the highest degree of certainty that a specified property belongs to each element of an infinite assemblage, an achievement to which the analytic, or syllogistic, method, dependent as it is upon the axioms of identity, contradiction and excluded third, is inadequate. For while these axioms validate deduction, inference from the general to the particular, it is precisely these, when regarded as the sole axioms of formal thought, which invalidate the inverse process. If this inverse process be not logically performable, then either mathematics neither is, nor

$\ddagger$ An interesting discussion of Poincaré's paper by G. Léchalas is found in the same volume of the same Revue. 
can become, a logically rigorous science or else it reduces in last analysis "à une immense tautologie."

Now the mathematician affirms the performability of the process in question, namely, in accordance with the argument form, complete induction. What, then, is, according to Poincaré, the logical ground of this affirmation?

We are first told what that ground is not. Suppose it established, in regard to some property $p:(1)$ that $p$ belongs to the number 1 ; $(2)$ that if $p$ belongs to an integer $n$, it belongs to $n+1$. Propositions (1) and (2) afford the means of generating one after another a sequence of syllogisms by which one proves first that $p$ belongs to 2 , then to 3 , and so on. In order to ascertain by this analytic method whether $p$ belongs to a specified integer $m$, it is necessary to determine in advance the same question for each of the integers $2,3, \cdots, m-1$, in the order as written, a process requiring a number of syllogisms which is greater the greater the number $m$. Accordingly, this method, of successive deductions, is not available for determining whether $p$ is a property of each in the totality of integers. Equally powerless to that end is experience (including observation) for this can take account of the individuals of a finite assemblage at most. Either analysis or experience may succeed if a sequence be finite but if it be infinite both must fail. Not less vain is it to invoke finally the aid of induction as employed in the physical sciences, for this latter, resting upon a purely assumed order in the external universe, is confessedly inductio imperfecta and as such can yield approximate certainty only.

Nevertheless, despite the inadequacy of the means mentioned, as soon as hypotheses (1) and (2) are granted and the indicated sequence of deductions is begun, "the judgment imposes itself upon us with irresistible evidence" that $p$ is a property of all the integers. Why? It appears to be clear that the answer must be the adduction of an additional presupposition of formal thought, a presupposition whose formulation shall mark a conscious extension of the domain of logic by affirming as axiomatic that apodictic certainty can transcend every limited sequence of deductions or observations. Such presupposition, which I venture to call the axiom of infinity, is stated by Poincaré, in answer to the foregoing question "why," as follows: "C'est qu'il n'est que l'affirmation de la puissance de l'esprit qui se sait capable de concevoir la répétition indéfinie d'un même acte dès que cet acte est une fois possible." 
Is the axiom sufficient? We are not explicitly told precisely what the operation (acte) is which in the present case it is at once possible and necessary to conceive as indefinitely repeated. To examine the matter, observe that, with a view to availing ourselves below of the familiar simplicity of the first Aristotelian figure, proposition (2) may be stated categorically as follows: every integer next after an integer having the property $p$ is an integer having the property $p$. Now denote by $P$ the expression : an integer having the property $p$; by $N$ the expression : integer next after $P$; and construct the pair of syllogisms

(i) $k$ is $P$,

(iii) every $N$ is $P$,

(ii) $k+1$ is an integer next after $k$,

(iv) $k+1$ is an $N$, $. \cdot k+1$ is an $N$; $. \cdot k+1$ is $P$.

(A) These syllogisms, being formally valid, will be valid no matter what the meaning of $k$, and will, therefore, be valid if $k$ be replaced by $k+1$. Such substitution yields a pair $\left(\alpha^{\prime}\right)$ of syllogisms which may be described as the pair next after the pair $(\alpha)$. The first premiss of $\left(\alpha^{\prime}\right)$ is the last conclusion of $(\alpha)$.

Let $k$ denote an integer, then (ii) is true by virtue of the assumed sequence of integers, and (iii) being true by (2), it is seen that the last conclusion of $(\alpha)$ is true provided the first premiss is true. By help of $(A)$ it follows that the last conclusion of $\left(\alpha^{\prime}\right)$ is true if the first premiss (i) of $(\alpha)$ is true, and the same holds for the last conclusion of $\left(\alpha^{\prime \prime}\right)$, pair next after $\left(\alpha^{\prime}\right)$, if such pair $\left(\alpha^{\prime \prime}\right)$ be supposed constructed, and so on ; i. e., if (i), first premiss of $(\alpha)$, be true, then will be true the last conclusions of all pairs of any sequence $(\alpha),\left(\alpha^{\prime}\right),\left(\alpha^{\prime \prime}\right), \ldots$, either actually or conceptually constructed. Now if $k=1$, then, by (1), (i) is true. Accordingly to gain certainty that every integer is $P$, it is sufficient to construct one after another a syllogistic pair for every integer, which is impossible, or to conceive it done, which is possible by axiom. Thus it is seen that the operation to which the axiom of infinity is to be applied in the present case is the operation $O$ (beginning with $(\alpha)$ for $k=1$ ) where $O$ denotes the construction of a syllogistic pair next after the pair last constructed. This conclusion appears to differ from that of Poincaré ${ }^{*}$ in that he appears to hold that

* Cf. op. cit., p. $379, \mathrm{~V}$. 
the operation in question is the construction of one syllogism instead of a pair. At all events, the operation is, in form, a deduction, and, for the following criticism, it is quite indifferent whether it be one or a pair.

The operation $O$ is obviously composite, and for the present purpose may be resolved into two operations $O_{1}$ and $O_{2}$ where $O_{1}$ (beginning with 1) means assigning to $k$ the integer next after the integer last assigned, and $\mathrm{O}_{2}$ (beginning with 1 ) means substituting for $k$ in $(\alpha)$ the (by $O_{1}$ ) assigned integer next after the integer last substituted in $(\alpha)$. Now of these component operations, the former is not deductive $(i . e$. , not " analytique," not syllogistic), while the latter is deductive, being a process of constructing arguments of type $(\alpha)$. The operation $\mathrm{O}_{2}$ presupposes $O_{1}$. The question arises: Is it necessary to apply the axiom of infinity to both $\mathrm{O}_{1}$ and $\mathrm{O}_{2}$ ? - a question to be answered in the following section.

\section{Examination of Dedekind's View.}

Let $S$ be a system of elements such that there is a law $\phi$ of depiction (Abbildung) depicting $S$ upon itself so that each element $e$ of $S$ is depicted upon one and but one element $e^{\prime}$ of $S$ and that no two elements are depicted upon a same element. Call $e^{\prime}$ the picture (Bild) or image of $e$. Every part of $S$ (including $S$ itself as a special case) thus depicted upon itself is named chain (Kette) under $\phi$. Denote by $A$ an arbitrary part of $S$ and by $A_{0}$ the assemblage of the elements common to all chains (in $S$ ) containing $A$. It is obvious that, $S$ and $\phi$ being granted, $A_{0}$ exists for every $A$, and Dedekind proves that $A_{0}$ is itself a chain, and describes it as the chain of $A$ under $\phi$. Let $\Sigma$ be an assemblage.

Theorem. - In order to prove that $A_{0}$ is part of $\Sigma$ it is sufficient to prove: $(\sigma)$ that $A$ is part of $\Sigma$, and $(\rho)$ that the image of every element of $A_{0}$ belonging to $\Sigma$ belongs to $\Sigma$.

An apparently simpler proof than that by Dedekind runs thus : Let $A_{0} \equiv A_{1}+A_{2}$ where $A_{1}$ denotes the assemblage of all those elements of $A_{0}$ that belong to $\Sigma$. By $(\rho), A_{1}$ is a chain, and, by $(\sigma)$, contains $A$. Hence, by definition of $A_{0}, A_{2}$ has no element, and $A_{0} \equiv A_{1}$.

Such is the beautiful theorem which the author characterizes - with what justification, we shall seek to determine at a later stage - as "die wissenschaftliche Grundlage" of complete induction. It affords the means of answering the closing ques- 
tion of II. If we denote by $S$ the assemblage of all integers, by $A$ the number 1 , and by $\Sigma$ the assemblage of all things each having the property $p$, then, the foregoing hypotheses (1) and (2) being granted, the proposition $p$ belongs to every integer, by Dedekind's theorem follows immediately on finding a $\phi$ (depicting $n$ on $n+1)$ under which $S \equiv A_{0}$ of 1 . Now precisely this identity is established by applying the axiom of infinity to the above defined operation $O_{1}$. Accordingly Poincarés application of the axiom to the analytic operation $\mathrm{O}_{2}$ is tautological. Indeed it is superfluous to use $\mathrm{O}_{2}$ even one time, and it appears that for Poincaré's problem the axiom might as well be reworded so as to restrict its application to $O_{1}$. So restricted, it is necessary, as seen, for Dedekind in the same connection. The supposed restriction, however, regarded as excluding $\mathrm{O}_{2}$, is rather apparent than genuine, for if one initially assumes, and this is possible without affecting the sufficiency of the application to $O_{1}$, that the $k$ of $O_{1}$ is in the diagrammatic scheme $(\alpha)$, then the application to $O_{1}$ carries with it the application to $O_{2}$. Next note that such deductive forms as $(\alpha)$ are presupposed by Dedekind, being in fact employed by him in proving his theorem. Accordingly, Dedekind's proof of the proposition, $p$ belongs to every integer, is not more fundamental than the application of Poincaré's axiom to $O_{2}$, and hence not more fundamental than Poincarés proof of the same proposition; than Poincarés "proof," I say, for while the application of his axiom to $\mathrm{O}_{2}$ is not necessary with, it is, we have seen, sufficient without, Dedekind's theorem - a fact which will presently be seen to be decisive against the latter author's claim of logical priority for his theorem. For we can now show that this theorem, so far from being "the scientific basis of," admits of being proved by, the method of mathematical induction. To this end we establish the

Theorem (a). - Every chain $A_{0}$ of a part $A$ of a system $S$ under $a \phi$ consists of a denumerable assemblage of assemblages

$$
A, A_{1}, A_{2}, \cdots, A_{n-\mathrm{I}}, A_{n}, A_{n+1}, \cdots
$$

where $A_{n}$ is the assemblage of those images of the elements of $A_{n-1}$ that are not in $A$.

For denote by $A_{1}$ the assemblage of elements that serve as images of all such elements of $A$ as are not imaged on elements of $A$. $A_{0}$, since it contains $A$ and is a chain, contains $A_{1}$. Denote by $A_{2}$ the assemblage of the elements that serve as 
images of those elements of $A_{1}$ that are not imaged on elements of $A$. It is plain that $A_{2}$ is in $A_{0}$, and, by definition of $\phi$, that no element is common to $A_{1}$ and $A_{2}$. The sequence of $A$ 's thus generable (or rather thus brought to attention one after another, for the $A$ 's are already generated by $\phi$ ) may or may not have an end. In either case the assemblage $E$ of all the elements in the $A$ 's obviously is a chain (in $S$ ) under $\phi$. The $A$ 's being in $A_{0}$, so is $E$; and $A$ being in chain $E$, so, by its definition, is $A_{0} ; . \cdot E \equiv A_{0}$.

Now assume Dedekind's data $(\sigma)$ and $(\rho)$. From $(\rho)$ follows $\left(\rho^{\prime}\right)$ : if $A_{n}$ belongs to $\Sigma$, then so does $A_{n+1}$. If now we regard $(\sigma)$ and $\left(\rho^{\prime}\right)$ as our data, then it follows, by Poincarés axiom, that every one of the $A$ 's and therewith $E\left(\equiv A_{0}\right)$ belongs to $\Sigma$. And so, it appears, the so-called foundation of ordinary mathematical induction is susceptible of being laid by ordinary mathematical induction.

In passing it may be noted that in the sequence of theorem (a) the power (in Cantor's sense) of none of the $A$ 's can be higher, though in each of those following some one it may be lower, than that of $A$. Accordingly, if we assume the proposition that the power of the assemblage of all the elements of the assemblages of a denumerable assemblage of assemblages having each of them a same power $\alpha$, is $\alpha$, then follows the

TheOREm (b).-The power of the chain $A_{0}$ of any given part $A$ of any given assemblage $S$ under a given $\phi$, is the same as the power of $A . *$

It is proper here to recognize a fact emphasized by Schröder $\dagger$ that Dedekind establishes his theorem without making use of either the notion of "number" or the notion of the number "series." On the other hand, the foregoing proof of the theorem by ordinary mathematical induction employs both these notions. But this difference does not justify the claim of relative fundamentality for that theorem, for the two notions mentioned, although they are by Dedekind introduced after, are not introduced through, the theorem, their being as concepts depending upon it neither mediately nor immediately.

Dedekind's theorem viewed as a generalization. Let $(\gamma)$ stand for the conclusion : $A_{0}$ belongs to $\Sigma$. Then the theorem, $T$, is : if $(\sigma)$ and $(\rho)$ are known, $(\gamma)$ can be inferred. In form this is

\footnotetext{
* The fact that $A$ may be finite and $A_{0}$ denumerably infinite is in spirit hardly an exception.

† Cf. op. cit., p. 355 .
} 
identical with that of mathematical induction as ordinarily understood. But the latter, call it $I$, deals with only a denumerable assemblage, $\phi$ depicting $n$ on the next, $n+1$; while $A_{0}$, subject of $T$, may have any power whatever (though Dedekind seems to think of no power higher than that of the continuum) and the image $e^{\prime}$ of $e$ may not be next to $e$. Accordingly $T$, dispensing with the notion of nextness essential to $I$, is a generalization of $I$. Now the $T$ data, $(\sigma)$ and $(\rho)$, are sufficient for $I$, for as we have seen, the $I$ data, $(\sigma)$ and $\left(\rho^{\prime}\right)$, are consequences of $(\sigma)$ and $(\rho)$. Accordingly, although $I$ is therefore available whenever $T$ is available, $T$ has nevertheless, especially in case $A_{0}$ is non-denumerable, a certain advantage over $I:(\gamma)$ is yielded immediately by $T$ but only mediately by $I$ (cf. theorem $(\alpha)$ ). On the other hand since $(\sigma)$ and $(\rho)$ are not consequences of $(\sigma)$ and $\left(\rho^{\prime}\right), I$ may be available when $T$ is not. So it appears that $T$, regarded as in the sense explained a generalization of $I$, is, as an engine of investigation, inferior to $I$.

The last conclusion hinges on the yet improved statement that $(\rho)$ is not a consequence of $\left(\rho^{\prime}\right)$. It will be sufficient to verify the statement by a simple example. Consider the assemblage $D$ of the elements $d$ of assemblages $D_{k}(k=1,2, \cdots)$. Suppose $D$ depicted upon itself by a $\phi$ so that every $d$ of $D_{n}$ is an image $d^{\prime}$ of one and but one $d$ of $D_{n-1}$ and that every $d^{n}$ of $D_{n-1}$ is imaged in either $D_{n}$ or $D_{1}$, but not in both. Under $\phi$, $D$ is obviously $D_{0}$ of $D_{1}$. For clearness we may suppose the $d$ 's to be delegates to a nominating convention. Now conceivably it may be known at once: $(\sigma)$ that every $d$ of $D_{1}$ will vote for $C ;\left(\rho^{\prime}\right)$ that every $d$ of $D_{n}$ will vote for $C$ if every $d$ of $D_{n-1}$ will do so ; and that if some $d$ will vote, not for $C$, either it is unknown how the $d^{\prime}$ of that $d$ will vote or that $d^{\prime}$ will vote, not for $C$; i. e., under $\phi$ the $I$ data $(\sigma)$ and $\left(\rho^{\prime}\right)$ are given while the $T$ hypothesis $(\rho)$ is either known to be false or not known to be true. Accordingly, if $\Sigma$ be the assemblage of $d$ 's who will vote for $C, I$ avails while $T$ fails to prove that $D$ belongs to $\Sigma$.

\section{Circularity of the Bolzano and Dedekind Proofs of the Ex- istence* Theorem for Infinite Assemblages.}

Bolzano's definition of infinite assemblage, introduced by aid of various subtile preliminaries $(\S \S 3-9), \dagger$ amounts to this : an

* For citation and analysis of the modern mathematical literature concerned with the question of actual infinity, cf. Veronese: Grundzüge der Geometrie. Note IV.

† Bolzano: Paradoxien des Unendlichen. 
assemblage is infinite if, and only if, it cannot be exhausted by removing from it, one after another, finite $(\S 8)$ assemblages of its elements. In $\S 13$, a proof is attempted of the proposition that such an assemblage exists, namely die Menge der Sätze und Wahrheiten an sich. The attempt informally postulates : the proposition, such truths exist, is such a truth, $A ; A$ is true, is another such truth, $B$; so on; and, the indicated process is inexhaustible. The last, an evident petitio principii, is doubtless to be granted, but only under some such axiom as that of Poincaré.

Bolzano affirms and exemplifies ( $(20)$, though he does not demonstrate, the proposition that every infinite assemblage can be paired in one-one fashion with a proper part of itself-a property employed independently by Dedekind as the defining property of the infinite and yielding a definition shown by Dedekind and independently by others * to be equivalent to the foregoing one of Bolzano's. By virtue of the "intrinsic" character of Dedekind's definition, his proof of the existence theorem better conceals, though it undoubtedly contains, a logical petitio. On examination the proof $\nmid$ is seen to postulate as certainties: (1) if there be a $t$, there is a $t^{\prime}$ (call it image of $t$ ) having $t$ as object ; (2) if there be two distinct $t$ 's, the corresponding $t^{\prime}$ 's are distinct; (3) there is a $t$; (4) there is a $t$ which is not a $t^{\prime} ;(5)$ every $t$ is another $t$ than its $t^{\prime}$. These being granted, it hardly follows deductively, though it is tacitly and, we may allow, admissibly $\S$ assumed, that there is an assemblage $\vartheta$ of $t$ 's, a totality excluding none of them. There is, then, plainly a $\phi$ depicting each $t$ on its $t^{\prime}$ and therewith, by virtue of (4), depicting $\vartheta$ on a proper part of itself;.$\ominus \vartheta$ is infinite. Now, provided one be permitted to reflect, it equally follows, from the postulates, that $\vartheta$ contains a sequence $S$ of $t^{\prime}$ s beginning with the $t$ of (4), each succeeding $t$ being the $t^{\prime}$ of its predecessor. $S$, too, is infinite by Dedekind's definition. Now certainty (1), in its character as a certainty postulated a priori, can not be contingent upon conclusions (such as that regarding $\vartheta$ or $S$ ) to be subsequently drawn from it joined to like certainties. Hence, even if the other postulates be rejected, certainty

* Cf. "Concerning positive definitions of finite assemblage, and infinite assemblage," BuLLETIN, vol. VII.

+ Cf. op. cit. , 866 .

$\neq$ The symbol $t$ standing for the word thought.

8 Such postulates as (1) and (2) seem to depend for their intelligibility upon the notion of totality. 
(1) involves certainty that the imaging process shall not fail even though as yet perhaps unknown considerations may demand that it be endlessly performable. Accordingly (1) involves a statement included in Poincaré's axiom, which appears indeed to be a presupposition of all logical discourse, the existence of the infinite being unavoidably however unconsciously assumed and so not demonstrable.

Columbia University.

\section{A GERMAN CALCULUS FOR ENGINEERS.}

Hauptsätze der Differential- und Integral-Rechnung. Von DR.

Robert Fricke. Dritte umgearbeitete Auflage. Braunschweig, Vieweg, 1902. 4vo., 218 pp.

WHILE the needs of American technical schools, and their environment, render a foreign book on the calculus unsuitable for use as a text, the difficult questions which arise in regard to the methods of presentation of this subject are largely the same throughout the world. It is a mistake to imagine that the German brain, for instance, is constructed so differently from the American, that the German Fuchs can grasp niceties of the calculus which necessarily escape the American Sophomore. Nor is it logical to presume that the tasks of an engineer differ materially in the two countries. The problems to be fought out are generally speaking about the same, aside from certain minor matters which depend upon traditional systems of instruction. The battle which is being waged on German soil for the closer union and more complete understanding between mathematicians and engineers, is therefore of almost equal interest to the same two classes in America. But we must here pass over the immense amount of fruitful material, which is the product of some of the most eminent minds of Germany*_among them Felix Klein - and which throws strong light on "the necessary and sufficient amount of calculus for the engineer." $\dagger$ It should be remarked, however, that the book which forms the subject of this review is produced, for use in a technical school, in the light of all this inspiring criti-

* See, e. g., the recent files of the Jahresbericht der Deutschen Mathematiker Vereinigung.

$\dagger$ Fricke, preface. 